\title{
Ethnobotany and Ethnopharmacology of the Americas
}

\author{
Lead Guest Editor \\ Myron Smith \\ Department of Biology, Carleton University, Ottawa, Ontario, Canada \\ Guest Editors \\ John Arnason \\ Department of Biology, University of Ottawa, Ottawa, Ontario, Canada
}

Alain Cuerrier

Jardin botanique de Montréal, Institut de recherche en biologie végétale, Université de Montréal, Montréal, Quebec, Canada 